\title{
Escala de actitudes hacia la natación en estudiantes universitarios. Validez y confiabilidad en estudiantes de educación física
}

Scale of attitudes towards swimming in university students. Validity and reliability in Physical Education students

\author{
José Damián Fuentes López \\ Universidad Nacional del Altiplano de Puno, \\ Instituto de Investigación en Ciencias de la Educación, \\ Perú jovican48@gmail.com
}

Juana Lucila Sánchez Macedo

Universidad Nacional del Altiplano de Puno,

Escuela Profesional de Educación Física, Perú

Eliseny Vargas Ramos

Universidad Nacional del Altiplano de Puno,

Escuela Profesional de Educación Física, Perú

Joel Adrián Begazo Armaza

Universidad Nacional del Altiplano de Puno,

Escuela Profesional de Educación Física, Perú

Angel Anibal Mamani Ramos

Universidad Nacional Mayor de San Marcos,

Escuela Profesional de Educación Física, Perú

\section{Resumen:}

La natación es una actividad acuática factible de ser aprovechada por los profesores de educación física como un medio formativo, en la región de Puno - Perú se practica con mayor frecuencia a pesar de sus condiciones climáticas adversas, por ello es importante fortalecer su aprendizaje en futuros enseñantes para enriquecer su perfil profesional; en consecuencia, la presente investigación buscó verificar validez y confiabilidad de la escala de actitudes hacia la natación (EAHN), en 192 estudiantes de la Escuela Profesional de Educación Física (EPEF) de la Universidad del Altiplano. Para la validez de contenido, a partir del análisis del dominio conceptual de la variable actitudes hacia la natación, se organizaron indicadores de seguridad, importancia, utilidad y predisposición; posteriormente se construyó escala con 31 preguntas, sometida a juicio de expertos que permitió obtener un coeficiente $\mathrm{V}$ de Aiken de 0.93 . La validez de constructo se realizó por el método multivariado de análisis factorial confirmatorio, cuyos resultados arrojaron saturaciones superiores a 0,35 para 30 preguntas. La confiabilidad se garantizó por medio de consistencia interna alpha de Cronbach con un índice de 0,86 para la escala en general, así también se determinó la estabilidad de la medición de los datos en el tiempo mediante la técnica test - retest con un coeficiente significativo de 0,81 . Se concluye afirmando que la escala es válida y confiable para estudiantes de la EPEF. Además, evidencian actitudes de aceptación a la natación.

Palabras Clave: Actitudes, Natación, Enseñanza, Validez, Confiabilidad.

\section{ABSTRACT:}

Swimming is an aquatic activity that can be used by Physical Education teachers as a training medium. In the region of Puno Peru, it is frequently practiced despite its adverse weather conditions, therefore it is important to strengthen its learning in future teachers to enrich their professional profile. Consequently, this investigation sought to verify the validity and reliability of the scale of attitudes towards swimming (EAHN), in 192 students of the Professional School of Physical Education (EPEF) of the Universidad del Altiplano. For content validity, based on the analysis of the conceptual domain of the variable attitudes towards swimming, indicators of safety, importance, usefulness and predisposition were organized. Subsequently, a scale was constructed with 31 questions, submitted to the judgment of experts, which allowed us to obtain an Aiken's $V$ coefficient of 0.93 . The construct

\section{Recepción: 17 Mayo 2020 | Aprobación: 20 Septiembre 2020 | Publicación: 30 Septiembre 2020}


validity was carried out by the multivariate method of confirmatory factor analysis, whose results showed saturations greater than 0.35 for 30 questions. Reliability was guaranteed by means of Cronbach's alpha internal consistency with an index of 0.86 for the scale in general, as well as the stability of the data measurement over time using the test - retest technique with a significant coefficient of 0.81. It is concluded that the scale is valid and reliable for EPEF students. In addition, they show acceptance attitudes towards swimming.

KeYwords: Attitudes, Swimming, Teaching, Validity, Reliability.

\section{A) INTRODUCCIÓN}

Para nadar es necesario el despliegue funcional de la totalidad del cuerpo sobre un soporte biológico y de habilidades, es relevante poseer capacidades de transformación personal y colectiva que configuran al nadador como un deportista completo (Campás, 2015), la natación como deporte consiste en desplazarse en el menor tiempo posible una distancia determinada gracias a la acción propulsora rítmica, repetitiva y coordinada de los miembros superiores, inferiores y el cuerpo que permitirá mantenerse en la superficie acuática y dominar la resistencia del agua para avanzar en ella (Palomino, González, Quiroga \& Ortega, 2015), por su carácter cíclico, caracterizado por la secuencia recurrente de movimientos durante el tiempo de nado, es recomendable el desarrollo de la flexibilidad en los hombros y tobillos para prevenir lesiones (Sanz, Martínez de Haro . Cid, 2016); la natación fue progresivamente construida para movilizarse en el agua evidenciando una determinada técnica con ahorro de energía y con implicancia funcional de procesos mentales que viabilizan la acción de nado, su aprendizaje desencadena en un cuerpo tecnificado (Castaño, 2014), su dominio evita ahogamientos (Contreras, 2011), el saber nadar se evidencia en la ejecución de uno o más estilos o técnicas natatorias con objetivos utilitarios y recreativos (Moreno \& Gutiérrez, 1998), para Bermúdez del Valle (1997) los objetivos de la natación se dan en función a planteamientos de necesidad utilitaria, higiénica, de salud, recreativa, competitiva y educativa que busca la adquisición y consolidación de patrones motrices acuáticos, la optimización de la condición física, el conocimiento y dominio progresivo de las técnicas de nado. El carácter utilitario radica en la necesidad primaria de sobrevivir o adaptarse al medio líquido y se evidencia desde las civilizaciones antiguas, (Campás, 2015) menciona como ejemplo la cueva de los nadadores del desierto del Líbano dónde se hallaron murales con hombres representando acciones de nado. El objetivo de salud de la natación ha sido el más documentado y ha demostrado beneficios de la natación que alcanzan la aptitud cardiorrespiratoria (Sánchez-Lastra, Martínez-Lemos, Díaz, Villanueva . Ayán, 2020), la composición corporal (Martínez-Sanz, Mielgo-Ayuso. Urdampilleta, 2012) y (Véliz, Maureira y Rodríguez, 2020); también encontramos resultados de reducción de la tensión arterial en adultos hipertensos (Da Silva, Pereira, Natali, Gomes de Souza y Dantas, 2006) y (Silva, Teixeira, Botelho, Dantas y Rama, 2015), igualmente es beneficiosa como tratamiento adicional al farmacológico para pacientes asmáticos (Rendón, Aguirre, Noroña, Betancourt, \& Vaca, 2017) y en la nueva forma de vida de pacientes con esclerosis múltiple (Bravo-Gonzales \& Álvarez-Roldán, 2019). En el plano psicológico se han encontrado evidencias de su aporte en la autoestima de personas mayores (Gallego, Aguilar, Cangas, Lorenzo, Franco y Mañas, 2012).

La enseñanza de la natación desde la niñez es importante para evitar la adquisición de fobias e inseguridades que obstaculizan su dominio, ésta debe implicar el aprendizaje de sus principios expresados a través de habilidades y destrezas en el agua (Sagarra, Gallego \& Monroy, 2017), en la misma vertiente Muñoz (2011) enfatiza la relevancia del medio acuático para el aprendizaje y adquisición de habilidades motrices que no surgen por maduración debido a su carácter ontogenético pero que son determinantes si se adquieren desde la primera infancia (Morales, 2010), consecuentemente, Sagarra et al., (2017) afirman que el aprendizaje de la natación requiere el dominio de sus principios expresados a través de habilidades y destrezas en el medio acuático para contrarrestar la disminuida ventaja adquirida genéticamente al ambiente acuático, y para ello se deben realizar técnicas respiratorias y movimientos propulsivos que garanticen la flotación dinámica (Gómez, 2012), algunos autores como Moreno \& Gutiérrez (1998) sostienen que el fin de la enseñanza de la natación 
son los estilos o técnicas natatorias. Para las personas adultas se deben aplicar metodologías diferentes de connotación andragógica, tomando en cuenta factores peculiares como la diversidad, los objetivos que persiguen, condiciones y/o limitaciones de capacidades biológicas, psicológicas y sociales (Negri, 2015). Las primeras formas metodológicas de enseñanza de la natación estuvieron orientadas a desarrollar la habilidad de desplazarse en el agua a través de acciones simultáneas y relacionadas entre imitación y progreso; con uso de flotadores naturales (Castaño, 2014), actualmente su enseñanza exige contemplar la aplicación de nuevas y pertinentes metodologías flexibles y exploratorias que combinen soluciones específicas además de ofrecer acciones de retroalimentación (Ruiz, 2017). En una perspectiva educativa de enfoque sociocultural la enseñanza de la natación debe superar paradigmas tradicionales homogenizantes basados en estrategias individualistas priorizadas para la competencia deportiva, que implican el uso de metodologías rutinarias, repetitivas y fragmentadas; por la naturaleza de sus diversos objetivos, la natación es producción cultural, en consecuencia, la enseñanza debe ser socializadora e inclusiva (Castaño, 2014).

La actitudes son variables importantes en el estudio de la psicología social, en el marco de la significancia social y cultural de la natación (anteriormente fundamentada), expresan formas de pensar sobre procesos y percepciones de estados psicológicos internos que orientan la conducta; por lo tanto, son predisposiciones aprendidas orientadas a reaccionar con niveles de valoración, aceptación o rechazo (Lima, Sáez, Cáceres \& Lima, 2013), como predisposiciones a la acción, las actitudes se han investigado en diferentes campos científicos que contribuyen a la comprensión del constructo, sus componentes cognitivos, afectivos y conductuales no son excluyentes y no necesariamente acogen todos los factores a la hora de su definición (Llorent \& Álamo, 2016), para Moreno (2006) las actitudes y valoraciones de los alumnos a las sesiones de aprendizaje son tema investigado en diferentes contextos y pueden ser positivas o negativas, por tanto, influyen en la adquisición de hábitos de práctica de ejercicio físico acuático, en consecuencia, es relevante validar instrumentos sobre actitudes hacia la natación, porque permiten proponer programas acuáticos diversos; esto contribuirá a una modificación positiva actitudinal, que predice la producción de una modificación positiva en los comportamientos (Laca, 2005).

En el contexto social de la enseñanza de la natación no se cuenta con instrumentos normalizados sobre actitudes hacia la natación, que permitan evaluar a grupos poblacionales ni mucho menos a futuros enseñantes, consecuentemente, ante la inexistencia de instrumentos válidos sobre actitudes a la natación, el presente estudio pretende validar la EAHN en estudiantes universitarios de educación física, afirmando hipotéticamente que la escala propuesta sería válida y confiable en una muestra de jóvenes universitarios peruanos que viven a elevada altitud del Perú (3.824 metros sobre el nivel del mar). Los objetivos del estudio son verificar la validez y confiabilidad de la EAHN y analizar la actitud de los estudiantes hacia la natación.

\section{в) Metodología}

\section{Ámbito o lugar del estudio}

El estudio se llevó a cabo en el departamento, provincia y distrito de Puno en la Universidad Nacional del Altiplano que está ubicada en la provincia y ciudad de Puno ubicado al extremo sur este del Perú. La Región abarca un perímetro fronterizo de $1108 \mathrm{Km}$. que representa el 11 por ciento de línea de frontera del Perú. Puno está a 3827 m.s.n.m. y sus principales ciudades con mayor índice poblacional estarían ubicados en un nivel de riesgo de altitud muy alta, que contempla altitudes entre 3500 a 5500 metros (Bernaola \& Ponce, 2012). 


\section{Descripción de métodos}

Se realizó un estudio descriptivo de corte transversal en estudiantes de la EPEF de Puno, cuya edad oscila entre 16,0 a 28,9 años. El tamaño representativo de la muestra fue calculado de forma probabilística (estratificada por ciclo de estudios), corresponde a 192 universitarios (56\%). Se incluyeron universitarios matriculados que asistieron el día de la evaluación. El estudio contó con la aprobación del comité de ética de la UNA.

\section{Procedimientos}

Para definir la edad cronológica se consideró la fecha de nacimiento y de evaluación de cada universitario (día, mes y año). Dos profesores recolectaron la información durante los meses de agosto y setiembre del 2019. Los estudiantes dispusieron de $30 \mathrm{~min}$ para responder a 31 interrogantes. El instrumento también fue aplicado en dos oportunidades al $12 \%$ de la población, con un intervalo de 7 días entre las dos mediciones (test y re-test).

\section{Instrumento}

Se construyó escala garantizada por validez de contenido a partir de la operacionalización de la variable denominada "actitudes hacia la natación", desagregada en cuatro indicadores: seguridad, importancia, utilidad y predisposición; con sus respectivos ítems de tipo cerrado, que en total fueron 31; el procedimiento se evidencia en la Tabla 1; la validez de contenido se garantizó mediante juicio de expertos, para esto se solicitó a cuatro doctores que laboran como docentes universitarios de Perú y uno del extranjero, total cinco; se buscó el completo acuerdo para cada ítem y los resultados se obtuvieron utilizando el coeficiente $\mathrm{V}$ de Aiken (Escurra, 1988), para la convocatoria y selección de jueces se consideró recomendación de (Pedrosa, Suárez \& García, 2013), en consecuencia todos contaban con perfil orientado al área de educación física y psicología, finalmente se les requirió apreciaciones con el objetivo de verificar pertinencia y calidad de cada ítem.

\section{B- Importancia:}

1. Para el desarrollo profesional de mi carrera considero que existen otras asignaturas más importantes que las de natación.

2. Para mi formación profesional las asignaturas más importantes que han de estudiarse tienen que ver con la natación.

3. Las actividades que se realizan en las clases de natación son poco interesantes.

4. La natación es importante para el desarrollo integral de la persona.

5. Practicar natación es saludable.

6. Es fundamental para mí desarrollar competencias para enseñar y entrenar en natación.

A- Seguridad:

1. Tengo temor a la asignatura de natación.

2. Tengo dificultades para nadar o desenvolverme con facilidad en una piscina u otro ambiente acuático.

3. Estoy calmado/a y tranquilo/a cuando ingreso a una piscina o ambiente acuático.

4. La natación hace que me sienta incómodo/a y nervioso/a.

5. Practicar natación no me asusta en absoluto.

6. Practicar natación hace que me sienta muy nervioso/a.

7. No me siento seguro cuando tengo que nadar en piscinas o ambientes acuáticos profundos. 
8. Me satisface nadar en piscinas u otros ambientes acuáticos.

9. Confío en mis habilidades acuáticas cuando ingreso a una piscina u otro ambiente acuático.

10. Prefiero usar flotadores para ingresar a una piscina profunda.

C- Utilidad:

1. Las asignaturas de natación pueden ser útiles solo para los que se dediquen exclusivamente a enseñar natación pero no para mí.

2. La natación no será de mayor utilidad en mi práctica como profesor de educación física

3. Considero la natación como una materia muy necesaria para mi profesión.

4. Saber nadar y enseñar a nadar incrementaría mis posibilidades de trabajo e ingresos económicos.

5. Puedo desarrollar actividades de enseñanza aprendizaje de la natación sin necesidad de saber nadar.

6. Nadar es divertido para mí.

7. Me gustaría tener una ocupación en la cual tuviera que enseñar a nadar.

8. Me gustaría tener una ocupación en la cual tuviera que entrenar nadadores.

9. Si tuviera oportunidad me inscribiría en más cursos de natación de los que son necesarios.

10. Las asignaturas de natación me desagradan.

D- Predisposición:

1. La natación es motivadora y estimulante para mí.

2. Quiero llegar a tener un conocimiento más profundo de la natación.

3. Espero tener que utilizar poco la natación en mi vida profesional.

4. Si me propondría llegaría a dominar la natación.

5. Me divierte el hablar con otros sobre natación.

\section{Análisis estadístico}

La normalidad de los datos se verificó por medio del test Kolmogorov-Smirnov. Se efectuó análisis estadístico descriptivo de frecuencias, porcentajes, media aritmética y desviación estándar. La validez de constructo se llevó a cabo por el método multivariado de análisis factorial confirmatorio A-FC (extracción de componentes principales), seguido de rotación Varimax y Kaiser-Meier-Olkin (KMO). Se determinó también las cargas factoriales (Saturaciones) y el porcentaje de la varianza.

La fiabilidad se garantizó por medio de consistencia interna (Alpha de Cronbach), así mismo, se determinó la estabilidad de los datos en el tiempo mediante la técnica test - retest, el coeficiente de Pearson muestra valor de (r) significativo equivalente a 0,81. Los cálculos estadísticos fueron efectuados en SPSS 25.0.

\section{C) Resultados y discusión}

La Tabla 2 describe las características de la muestra, se observa hábitos de beber con frecuencia a veces en un $50 \%$ y de fumar $40 \%$. El 51\% proceden de zonas de vivienda rurales, el $95.8 \%$ nacieron en Puno y las provincias de mayor procedencia son Puno, Azángaro y Melgar. 
TABLAS 2

Variables que caracterizan la muestra

\begin{tabular}{|c|c|c|c|}
\hline \multirow{2}{*}{ Variables } & \multicolumn{2}{|c|}{$(n=199)$} & \multirow{2}{*}{$\mathbf{p}$} \\
\hline & $\mathrm{x}$ & $\mathrm{DE}$ & \\
\hline Edad & 20,5 & 2,9 & 0,000 \\
\hline Actitud a la natación & 114,9 & 16,8 & 0,000 \\
\hline Seguridad & 35,6 & 7,2 & 0,000 \\
\hline Importancia & 23,5 & 4,2 & 0,000 \\
\hline Utilidad & 16,3 & 2,2 & 0,000 \\
\hline Predisposición & 39,5 & 7,4 & 0,000 \\
\hline Sexo & $\mathbf{N}$ & $\%$ & \\
\hline Hombre & 163 & 84,9 & \\
\hline Mujer & 29 & 15,1 & 0,000 \\
\hline \multicolumn{4}{|l|}{ Hábitos de fumar } \\
\hline a) Siempre & 1 & 0,5 & \multirow{3}{*}{0,000} \\
\hline b) A veces & 40 & 20,8 & \\
\hline c) Nunca & 151 & 78,6 & \\
\hline \multicolumn{4}{|l|}{ Hábitos de beber } \\
\hline a) Siempre & 3 & 1,6 & \multirow{3}{*}{0,000} \\
\hline b) A veces & 96 & 50,0 & \\
\hline c) Nunca & 93 & 48,4 & \\
\hline \multicolumn{4}{|l|}{ Zona de vivienda } \\
\hline a) Rural & 98 & 51.0 & \multirow[b]{2}{*}{0,000} \\
\hline b) Urbana & 94 & 49.0 & \\
\hline \multicolumn{4}{|c|}{ Lugar de nacimiento (región) } \\
\hline $\begin{array}{l}\text { Puno } \\
\text { Pute }\end{array}$ & 184 & 95,8 & \multirow{4}{*}{0,000} \\
\hline Arequipa & 5 & 2,6 & \\
\hline Cusco & 2 & $\begin{array}{l}2,0 \\
1,0\end{array}$ & \\
\hline Lima & 1 & 0,5 & \\
\hline \multicolumn{4}{|c|}{ Lugar de nacimiento (provincia) } \\
\hline Huancané & 8 & 4,2 & \multirow{14}{*}{0,000} \\
\hline Chucuito & 14 & 7,3 & \\
\hline Sandia & 6 & 3,1 & \\
\hline Melgar & 20 & 10,4 & \\
\hline Lampa & 10 & 5,2 & \\
\hline Azángaro & 21 & 10,9 & \\
\hline Puno & 47 & 24,5 & \\
\hline Collao & 9 & 4,7 & \\
\hline Carabaya & 20 & 10,4 & \\
\hline San Román & 11 & 5,7 & \\
\hline San Antonio de Putina & 6 & 3,1 & \\
\hline Moho & 5 & 2,6 & \\
\hline Yunguyo & 5 & 2,6 & \\
\hline Provincias de otra región & 10 & 5,2 & \\
\hline
\end{tabular}

El análisis de pertinencia del ítem con el indicador y claridad en la redacción del ítem se realizó con la $\mathrm{V}$ de Aiken en 31 elementos, en la Tabla 3 se observa una $V$ de Aiken equivalente a 0,94 para el indicador de seguridad, 0.93 para el indicador de importancia, para utilidad equivale a 0.95 y para el indicador de predisposición 0.91; la $\mathrm{V}$ de Aiken total asciende a 0.93.

Tabla 3. Resultados de validez de contenido mediante coeficiente $V$ de Aiken 
TABLA 3

Resultados de validez de contenido mediante coeficiente $V$ de Aiken

\begin{tabular}{|c|c|c|c|c|c|c|}
\hline Indicador & Pertinencia & Claridad & Promedio & Ítems & Pertinencia & Claridad \\
\hline \multirow{10}{*}{ Seguridad } & \multirow{10}{*}{0,93} & \multirow{10}{*}{0,95} & \multirow{10}{*}{0,94} & 1 & 0,95 & 0,95 \\
\hline & & & & 2 & 1,00 & 1,00 \\
\hline & & & & 3 & 0,95 & 1,00 \\
\hline & & & & 4 & 1,00 & 0,90 \\
\hline & & & & 5 & 0,95 & 1,00 \\
\hline & & & & 6 & 0,90 & 0,95 \\
\hline & & & & 7 & 0,95 & 0,95 \\
\hline & & & & 8 & 0,80 & 0,95 \\
\hline & & & & 9 & 0,90 & 0,90 \\
\hline & & & & 31 & 0,90 & 0,85 \\
\hline \multirow{6}{*}{ Importancia } & \multirow{6}{*}{0,93} & \multirow{6}{*}{0,93} & \multirow{6}{*}{0,93} & 10 & 0,95 & 0,90 \\
\hline & & & & 11 & 0,95 & 1,00 \\
\hline & & & & 12 & 0,95 & 0,90 \\
\hline & & & & 13 & 0,90 & 0,85 \\
\hline & & & & 14 & 0,90 & 1,00 \\
\hline & & & & 15 & 0,90 & 0,95 \\
\hline \multirow{5}{*}{ Utilidad } & \multirow{5}{*}{0,96} & \multirow{5}{*}{0,94} & \multirow{5}{*}{0,95} & 16 & 0,95 & 0,90 \\
\hline & & & & 17 & 1,00 & 1,00 \\
\hline & & & & 18 & 0,95 & 0,95 \\
\hline & & & & 19 & 0,95 & 0,90 \\
\hline & & & & 20 & 0,95 & 0,95 \\
\hline \multirow{10}{*}{ Predisposición } & \multirow{10}{*}{0,90} & \multirow{10}{*}{0,93} & \multirow{10}{*}{0,91} & 21 & 1,00 & 0,95 \\
\hline & & & & 22 & 0,90 & 1,00 \\
\hline & & & & 23 & 0,90 & 0,80 \\
\hline & & & & 24 & 0,90 & 1,00 \\
\hline & & & & 25 & 0,90 & 1,00 \\
\hline & & & & 26 & 0,90 & 0,90 \\
\hline & & & & 27 & 0,90 & 0,90 \\
\hline & & & & 28 & 0,85 & 0,80 \\
\hline & & & & 29 & 0,85 & 0,90 \\
\hline & & & & 30 & 0,90 & 1,00 \\
\hline EAHN (Total) & 0,92 & 0,94 & 0,93 & 0,93 & & \\
\hline
\end{tabular}

En la Tabla 4 se observa el valor de $\mathrm{KMO}=0,897>0,500$. La prueba de Bartlett tiene una $\mathrm{p}=0,000<0,050$ que permite rechazar la hipótesis de matriz identidad. El diagnóstico es positivo, es decir que se cumplieron satisfactoriamente las condiciones para aplicar el análisis factorial.

TABLA 4

Prueba de KMO y Bartlett Esta va sin imagen

\begin{tabular}{lll}
\hline Medida Kaiser-Meyer-Olkin de adecuación de muestreo & 0,897 \\
Prueba de esfericidad & Aprox. Chi-cuadrado & 2686,15 \\
de Bartlett & gl & 465 \\
& Sig. & 0,000 \\
\hline
\end{tabular}

El análisis factorial evidencia cuatro factores en 31 ítems. Los valores de saturación son superiores a 0,35 excepto el ítem 11. El porcentaje de explicación de todo el instrumento ascendió a 50,78\%, tal como se muestra en la Figura 1, en general, la EAHN mostró un elevado nivel de consistencia interna reflejado con un índice alfa de Cronbach de 0,867. 
FIGURA 1

Valores del análisis confirmatorio y alfa de Cronbach si se elimina el elemento

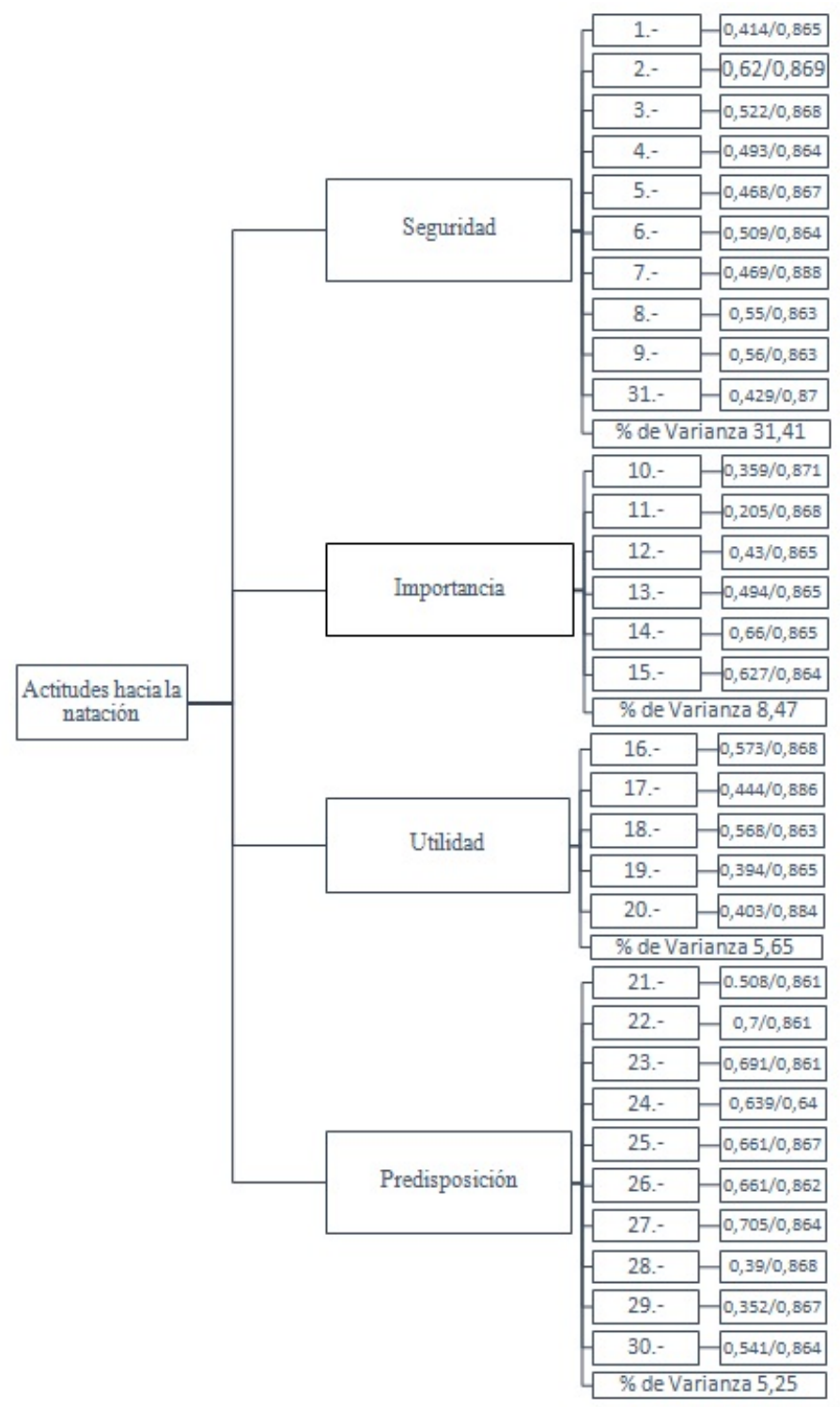

Elaboración propia

En la Tabla 5 se presentan datos agrupados por indicadores y en general sobre la actitud a la natación, se puede advertir en todos los casos que los estudiantes en forma mayoritaria muestran actitudes de aceptación, en cuanto a la diferencia de género no existen mayores diferencias en los indicadores, en el resultado global de la escala se observa una ligera supremacía que favorece a los hombres. 
TABLA 5

Actitud hacia la natación

\begin{tabular}{lllllllll}
\hline \multirow{2}{*}{ Indicadores } & \multirow{2}{*}{ Rango } & \multirow{2}{*}{ Actitud } & \multicolumn{2}{l}{ Hombre } & \multicolumn{2}{l}{ Mujer } & \multicolumn{2}{c}{ Ambos } \\
& & & $\mathrm{F}$ & $\%$ & $\mathrm{~F}$ & $\%$ & $\mathrm{~F}$ & $\%$ \\
\hline \multirow{3}{*}{ Seguridad } & $<=25$ & Rechazo & 15 & 9.2 & 3 & 10.3 & 18 & 9.4 \\
& $26+$ & Aceptación & 148 & 90.8 & 26 & 89.7 & 174 & 90.6 \\
Utilidad & $<=13$ & Rechazo & 8 & 4.9 & 1 & 3.4 & 9 & 4.7 \\
& $14+$ & Aceptación & 155 & 95.1 & 28 & 96.6 & 183 & 95.3 \\
Importancia & $<=15$ & Rechazo & 6 & 3.7 & 1 & 3.4 & 7 & 3.6 \\
& $16+$ & Aceptación & 157 & 96.3 & 28 & 96.6 & 185 & 96.4 \\
Predisposición & $<=25$ & Rechazo & 8 & 4.9 & 2 & 6.9 & 10 & 5.2 \\
& $26+$ & Aceptación & 155 & 95.1 & 27 & 93.1 & 182 & 94.8 \\
General & $<=102$ & Rechazo & 37 & 22.7 & 9 & 31.0 & 46 & 24.0 \\
& $103+$ & Aceptación & 126 & 77.3 & 20 & 69.0 & 146 & 76.0 \\
\hline
\end{tabular}

\section{Discusión}

La natación es factible de practicar sin tomar en cuenta la edad debido al bajo impacto sobre las distintas partes del cuerpo que reducen la tensión de los huesos y articulaciones, además de beneficiar aspectos psíquicos, físicos y fisiológicos que se expresarán en mayor bienestar y autonomía personal (Gallego, J., Aguilar, J., Cangas, A., Lorenzo, J., Franco, C., y Mañas, 2012), ha sido constantemente afectada por el determinismo cultural, sin embargo, su investigación va más allá de describir aspectos biomecánicos que revelan formatos metodológicos para su enseñanza (Castaño, 2014), el objetivo de la presente investigación fue verificar la validez y confiabilidad de la EAHN y su respectiva tendencia de aceptación o rechazo a la natación, para ello en un primer momento se garantizó con validez de contenido a partir de la operacionalización de la variable denominada actitudes hacia la natación, los principales indicadores de la variable fueron seguridad, importancia, utilidad y predisposición; la seguridad relacionada con la confianza del estudiante al ingresar a ambientes acuáticos, la importancia de la asignatura de natación en el plan de estudios para lograr el perfil del estudiante, la utilidad de la natación para el desempeño laboral y la predisposición a la enseñanza y aprendizaje de la natación. Dicha validez de contenido se realizó mediante cinco expertos, de forma similar a otros estudios (Nieves, Mayora y Ojeda, 2013); (López, Lalangui, Maldonado y Palmero, 2019); (Oñate, Batalla y Paez, 2020), los resultados cualitativos se cuantificaron haciendo uso de la $\mathrm{V}$ de Aiken, el coeficiente global de pertinencia y claridad de los ítems equivale a 0.93, éste es aceptable debido a que evidencia acuerdo de consenso en el marco de lo sugerido por (Escurra, 1988), la metodología desarrollada se alinea con estudios que utilizaron similar metodología para dar validez de contenido a instrumentos sobre actitudes (Dorantes, Hernández y Tobón, 2016); (Aldana de Becerra, Caraballo y Babativa, 2016); (Ingunza y Carrasco, 2019). Para la validez de constructo los valores obtenidos en el análisis factorial confirmatorio arrojaron saturaciones superiores a la referencia mínima para retener ítems, que fue 0.35 (López, R., Avello, R., Palmero, D., Sánchez, S., y Quintana, M., 2019), los resultados son coherentes con estudios que aplicaron análisis factorial confirmatorio (Cossio, M., Vasquez, P., y Luarte, C., 2016); (Gómez, Fuentes, Canqui, de Arruda, y Cossio, 2016); (Aguilar, Marqués, Serra, Dueñas y Sempere, 2017). El coeficiente alfa de Cronbach que estima la consistencia interna total del instrumento (Cronbach \& Meehl, 1955), fue equivalente a 0,86, los valores para cada ítem en caso de ser eliminado son superiores a 0,86 , esto garantiza la confiabilidad de las mediciones que son similares a otras investigaciones (Baca, 2016); (Pedraza et al., 2016) y (Albarrán, V., Geldres, V., Paredes, P., Ramirez, O., Ruiz, F., y Palomino, J. C., 2018).

Finalmente se puede advertir en todos los casos o indicadores que los estudiantes en forma mayoritaria, muestran actitudes de aceptación, los datos se agruparon en actitudes de rechazo y aceptación; y los resultados evidencian que el 76\% de estudiantes evidencian actitudes de aceptación hacia la natación, este resultado es 
similar al de (Carcamo, Wydra, Hernandez y Martinez, 2017), pero sobre todo guarda coherencia con una concepción integral de la natación establecida por Castaño (2014) en el sentido de emancipar la natación del fenómeno deportivista. Es importante señalar que los estudiantes investigados abrigan el deseo de ser profesores de educación física y estudian para ello, proceden de diversas provincias y distritos de la región puneña que está ubicada en un nivel de riesgo de altitud (Bernaola y Ponce, 2012) y que en algunos casos superan el 66\% de pobreza (Ministerio de Desarrollo e Inclusión Social, 2019); en este contexto, la práctica de la natación es seriamente limitada y a veces inexistente en la mayoría de las provincias, sobre todo por factores climatológicos, económicos y de infraestructura; lo cual hace que los estudiantes en su mayoría posean mínimo desarrollo de habilidades motrices en ambientes acuáticos. Sin embargo, el nivel alto de aceptación a la natación encontrado en el presente estudio puede ser explicable en el marco de los componentes cognoscitivos, afectivos y conductuales que integran las actitudes (Montes, Alcántar, Padilla y Pulido, 2015), por tanto, intervinieron otros factores socio culturales que condicionan su presencia o ausencia.

En el indicador de seguridad se observó que el 90\% de estudiantes muestran actitud de aceptación, el resultado hallado implicaría la mínima existencia de temor y es consonante con lo señalado por Guerra, J., Gutiérrez, M., Zavala M., Singre, J., Goosdenovich Campoverd, D. \& Romero, E. (2017) quienes afirman que la práctica de una natación segura podría permitir incremento de confianza de las personas; en la misma línea Sagarra et al., (2017) enfatizan su enseñanza desde la infancia para evitar la adquisición de inseguridades. Por lo tanto, la seguridad en el estudiante es una fortaleza para su aprendizaje, este se da sobre todo en actividades físicas o deportivas como la natación que presentan algún riesgo, para esto también es pertinente el aporte de Contreras (2011) al recomendar el tipo de alberca; por otro lado, el resultado es coherente con la edad avanzada de los estudiantes que guarda relación con aptitudes emocionales y motivacionales (Musch \& Grondin 2001), en cuanto a la utilidad el 95\% presentó actitud de aceptación, que está referida a la utilidad de la natación para el futuro profesor como una posibilidad de desempeño docente y se relacionaría con aspectos metodológicos, estos resultados son similares a los hallados por (Balderrama-Ruedas, DíazDomínguez y Gómez-Castillo, 2014) en alumnas futuras docentes con elevada aceptación a la natación. El indicador de importancia se evidencia con un $96.4 \%$ de aceptación, este resultado es coherente con estudios como el de (García, Robles \& Morilla, 2005) que evidenciaron la importancia de la natación para la mejora de la salud y recomiendan realizar tres sesiones semanales; Contreras (2011) que considera la natación como deporte completo y divertido u Ordoñez \& Salazar (2015) que comprobaron la efectividad de un programa de actividades acuáticas en el mejoramiento del desarrollo psicomotor. La predisposición nos evidencia un 95\% de aceptación, entendida esta como una disposición anticipada a nivel psicológico y mental, Balderrama et al., (2014) mostraron resultados similares de alta predisposición a la práctica y aprendizaje de la natación.

\section{D) Conclusiones}

A partir de los resultados obtenidos se afirma que la escala de actitudes hacia la natación para estudiantes universitarios de la EPEF es válida, ésta se garantizó a través del análisis del dominio conceptual de la variable que permitió definir el instrumento en cuatro indicadores de seguridad, importancia, utilidad y predisposición, y en consecuencia construir la escala con 31 interrogantes que fueron sometidos a juicio de expertos obteniendo un adecuado coeficiente $\mathrm{V}$ de Aiken. Finalmente, con la aplicación del análisis factorial confirmatorio se ratificaron 30 ítems o cuestiones debido a que sólo el ítem 11 presentó saturación baja.

La confiabilidad del instrumento se garantizó por medio de consistencia interna alpha de Cronbach con un índice aceptable para la escala en general, del mismo modo se determinó la estabilidad de la medición de los datos en el tiempo mediante la técnica test - retest.

Los estudiantes universitarios muestran actitudes de aceptación hacia la natación, de la misma forma que en los indicadores de seguridad, importancia, utilidad y predisposición; esto se dio a pesar de 
existir limitaciones climatológicas, económicas y de infraestructura para la práctica acuática; sin embargo, intervienen otros factores socio culturales que condicionan actitudes de aceptación.

Consideramos algunas limitaciones como el hecho de que son casi inexistentes estudios similares que permitan realizar comparación concreta de resultados, éstos, generan la necesidad de investigar posibles factores ambientales y vocacionales que condicionan actitudes favorables hacia la natación en futuros docentes de educación física.

\section{CONFLiCTO DE INTERESES}

Los autores declaran no tener ningún conflicto de intereses.

\section{Agradecimientos}

Nuestra gratitud a los estudiantes del X Semestre de la Escuela Profesional de Educación Física.

\section{Referencias Bibliográficas.}

Albarrán, V., Geldres, V., Paredes, P., Ramirez, O., Ruiz, F., y Palomino, J. C. (2018). Validación de la batería de instrumentos para la evaluación de factores de riesgo psicosocial. Horizonte Médico-Lima, 18(1), 59 - 67. https ://doi.org/10.24265/horizmed.2018.v18n1.09

Aldana de Becerra, G., Caraballo, G., y Babativa, D. (2016). Escala para Medir Actitudes hacia la Investigación (eacin): validación de contenido y confiabilidad. Revista Aletheia, 8(2), 104-121. Disponible en https://aletheia.cinde.o rg.co/index.php/ALETHEIA/article/view/325/253

Aguilar, M., Marqués, E., Serra, P., Dueñas, L., y Sempere, N. (2017). Elaboración y validación del «Cuestionario de actitudes hacia la ética profesional en Fisioterapia». Fisioterapia, 39(4), 148-157. https://doi.org/10.1016/j.ft .2016.12.001

Baca, R. (2016). Confiabilidad y validez del cuestionario de apoyo social en pacientes con cáncer de Trujillo. Revista de Investigación En Psicología, 19(1), 177. https://doi.org/10.15381/rinvp.v19i1.12452

Balderrama-Ruedas, J. A., Díaz-Domínguez, P. J. y Gómez-Castillo, R. I. (2014). Actitudes hacia la actividad física y el deporte. Ra Ximhai, 10(5), 339-352. https://www.redalyc.org/articulo.oa?id=461/46132134022

Bermúdez del Valle, A. (1997). La natación en la escuela. En F. Giménez, P. Sáenz-López, y M. Díaz (Comp.), El deporte escolar (pp. 85-94). Huelva: Universidad de Huelva.

Bernaola, M. y Ponce, J. (2012). Sección Técnica. Los riesgos de la altitud y su prevención. Seguridad y Salud En El Trabajo, 68,6-12.http://ergopar.istas.net/ficheros/documentos/Seguridad_y_Salud_trabajo_68.INSHT.Exp eriencias.pdf

Bravo-González, F. y Álvarez-Roldán, A. (2019). Esclerosis múltiple, pérdida de funcionalidad y género. Gaceta Sanitaria, 33(2), 177-184. https://10.1016/j.gaceta.2017.09.010

Campás, G. P. (2015). Sobre mitos de la natación competitiva y la natación educativa: una histórica y cultural desde Elías Juncosa. Materiales para la Historia del Deporte, 13, 78. http://polired.upm.es/index.php/materiales_his toria_deporte/article/view/4116

Carcamo, J., Wydra, G., Hernandez, C., y Martinez, C. (2017). Actitudes hacia la educación física: Grados de importancia y conformidad según escolares de Chile y Alemania. Una mirada intercultural. RETOS, 2041(32), 158-162. https://recyt.fecyt.es/index.php/retos/article/view/52824

Castaño Marín, J. C. (2014). La natación: arqueología y genealogía de una práctica [en línea]. Tesis de posgrado. Universidad Nacional de la Plata. Facultad de Humanidades y Ciencias de la Educación. Disponible en: https: //doi.org/10.35537/10915/42989 
Contreras, C. (2011). La importancia de la práctica de la natación en la educación de los niños y las niñas. EF Deportes Revista Digital 16(161), 1-7. Disponioble en http://www.efdeportes.com/efd161/la-importancia-de-la-practic a-de-la-natacion.htm

Cossio, M., Vasquez, P., y Luarte, C. (2016). Evaluación de la autopercepción de la aptitud física y propuesta de normativas en adolescentes escolares chilenos: estudio EAPAF. Archivos Argentinos de Pediatria, 114(04), 319328. https://doi.org/10.5546/aap.2016.319

Cronbach, L. J., \& Meehl, P. E. (1955). Construct Validity in psychological Tests. Boletín Psicológico, 52(4), 281-302. https://doi.org/https://doi.org/10.1037/h0040957

Da Silva, J., Santos, J., Natali, A., Gomes de Souza, R. y Martín, E. (2006). Efectos crónicos de un programa regular de natación. Sobre la tensión arterial de adultos hipertensos. RICYDE. Revista internacional de ciencias del deporte, 2(4), 15-24. https://10.5232/ricyde2006.00402.

Silva, J., Teixeira, A., Botelho, A., Dantas \& Rama, L. (2015). Comportamento Da pressão arterial em homens pré-hipertensos participantes em um programa regular de natação. Revista Brasileira de Medicina do Esporte, 21(3), 178-181. https://10.1590/1517-8692201521032115. (Error 1: El enlace externo https:// 10.1590/1517-8692201521032115 debe ser una URL) (Error 2: La URL https:// $10.1590 / 1517-8692201521032115$ no esta bien escrita)

Dorantes, J., Hernández, J., y Tobón, S. (2016). Juicio de expertos para la validación de un instrumento de medición del síndrome de burnout en la docencia. Ra Ximhai, 12(6), 327-346. https://doi.org/10.35197/rx.12.01.e3.2 016.22.jd

Escurra, L. (1988). Cuantificación de la validez de contenido por criterio de jueces. Revista De Psicología, 6(1-2), 103111. http://revistas.pucp.edu.pe/index.php/psicologia/article/view/4555

Gallego, J., Aguilar, J., Cangas, A., Lorenzo, J., Franco, C., y Mañas, I. (2012). Programa de Natación Adaptada para Personas Mayores Dependientes: Beneficios Psicológicos , Físicos y Fisiológicos. Revista de Psicología Del Deporte, 21(1), 125-133. https://www.redalyc.org/pdf/2351/235124455016.pdf

García, J., Robles, F. y Morilla, J. (2005). Análisis comparativo de los beneficios de la natación en jóvenes adolescentes en relación del número de sesiones semanales. http://www.munideporte.com/imagenes/documentacion/fiche ros/20100608130300jose_antonio_garcia.pdf

Gómez, R., Fuentes, J., Canqui, L., de Arruda, M. y Cossio, M. (2016). Reproductibilidad de un cuestionario que valora la actividad física en adolescentes escolares de altitud. Salud Uninorte, 32(1), 95-104. https://doi.org/10.1448 2/sun.32.1.8477

Gómez, J. (2012). Enseñanza de los estilos de natación crol, espalda, mariposa y braza. Madrid: Wanceulen.

Guerra, J., Gutiérrez, M., Zavala M., Singre, J., Goosdenovich Campoverd, D. \& Romero, E. (2017). Relación entre ansiedad y ejercicio físico. Revista Cubana de Investigaciones Biomédicas, 36(2), 169-177. http://scielo.sld.cu/sc ielo.php?script=sci_arttext\&pid=S0864-03002017000200021\&lng=es\&tlng=es.

Ingunza, N. y Carrasco, M. (2019). Validez y confiabilidad del cuestionario de interacción trabajo-familia (swing) en trabajadores de una empresa minera de la libertad, Perú. Revista Boletín Redipe, 8(8), 144-152. https://doi.or g/10.36260/rbr.v8i8.807

Laca, F. (2005). Actitudes y comportamientos en las situaciones de conflicto. Enseñanza e Investigación En Psicología, 10(1), 117-126. https://www.redalyc.org/pdf/292/29210108.pdf

Lima, M., Sáez, A., Cáceres, B., y Lima, J. (2013). Diseño y validación de escalas para medir la actitud adolescente hacia: Sexualidad, sustancias adictivas y seguridad vial. ¿se relacionan con los comportamientos?. Anales Del Sistema Sanitario de Navarra, 36(2), 203-215. https://doi.org/10.4321/s1137-66272013000200004

Llorent, V. J., y Álamo, M. (2016). Escala de actitudes hacia la diversidad cultural (ADC) para los futuros docentes. Opcion, 32(11), 832-841. https://www.redalyc.org/articulo.oa?id=31048902048

López, R., Lalangui, J., Maldonado, A., y Palmero, D. (2019). Validación de un instrumento sobre los destinos turísticos para determinar las potencialidades turísticas en la provincia de El Oro, Ecuador. Universidad y Sociedad, 11(2), 341-346. http://rus.ucf.edu.cu/index.php/rus 
López, R., Avello, R., Palmero, D., Sánchez, S., y Quintana, M. (2019). Validación de instrumentos como garantía de la credibilidad en las investigaciones científicas. Revista Cubana de Medicina Militar, 48(2), 0-10. http://www .revmedmilitar.sld.cu/index.php/mil/article/view/390/331

Martínez-Sanz, J. M., Mielgo-Ayuso, J. y Urdampilleta, A. (2012). Composición corporal y somatotipo de nadadores adolescentes federados. Revista Española de Nutrición Humana y Dietética, 16(4),130-136. http://dx.doi.org/1 0.14306/renhyd.16.4.59

Ministerio de Desarrollo e Inclusión Social. (2019). Reporte Regional de Indicadores Sociales del Departamento de Puno. http://sdv.midis.gob.pe/Sis_Anemia/Uploads/Indicadores/PUNO.pdf

Montes, J., Alcántar, M., Padilla y Pulido, I. (2015). Actitudes de los estudiantes universitarios ante la tutoría. Diseño de una escala de medición. Revista de La Educacion Superior, 44(173), 103-124. https://doi.org/10.1016/j.res u.2015.04.004

Morales, E. (2010). Propuesta educativa «El agua como medio de enseñanza: importancia de la evaluación».RETOS. Nuevas Tendencias en Educación Física, Deporte y Recreación, 17,72-75. https://www.redalyc.org/articulo.oa?id $=3457 / 345732283015$

Moreno, J. (2006). Actitudes hacia la práctica físico-deportiva según el sexo del practicante. International Journal of Sport Science, 2(3), 20-43. https://doi.org/10.5232/ricyde2006.003.02

Moreno, J., Gutierrez, M. (1998). Bases metodológicaspara el aprendizaje de las actividades acuáticas educativas. Madrid: INDE.

Musch, J. \& Grondin, S. (2001). Unequal competition as an impediment to personal development: A review of the relative age effect in sport. Developmental review, 21(2),147-67. https://doi.org/10.1006/drev.2000.0516

Muñoz, E. (2011). La educación integral y la motivación en las actividades acuáticas: Un estudio cientifico aplicado. Madrid: Wanceulen.

Negri, D. (2015). Natación en la adultez. Un espacio donde se logra algo más que aprender a nadar. $11^{\circ}$ Congreso Argentino de Educación Física y Ciencias, 28 de septiembre al 10 octubre de 2015, Ensenada, Argentina. En Memoria Académica. http://www.memoria.fahce.unlp.edu.ar/trab_eventos/ev.7273/ev.7273.pdf

Nieves, I., Mayora, C., y Ojeda, V. (2013). Validación por juicio de expertos de un prototipo de software de lectura extensiva en inglés asistida por el computador. Investigacion y Postgrado, 28(2), 9-35. http://ve.scielo.org/sciel o.php?script $=$ sci_arttext\&pid $=$ S1316-00872013000200002

Oñate, C., Batalla, A. y Paez, J. (2020). Elaboración y validez de un cuestionario de las habilidades motrices iniciales para estudiantes de enseñanza media. Retos, 38, 465-471. https://doi.org/10.47197/retos.v38i38.75537

Ordóñez, K. Y Salazar, D. (2015). Estudio de los beneficios de la natación en el desarrollo psicomotor en niños de entre 7-11 años de la unidad educativa Victor Gerardo Aguilar, en el año 2014. Tesis de pregrado. Facultad De Filosofía, Letras y Ciencias de la Educación. Carrera de Cultura Física. Ecuador.

Palomino, A., González, V., Quiroga, M., y Ortega, F. (2015). Efectos del Entrenamiento de Natación sobre la Asimetría Corporal en Adolescentes. International Journal of Morphology, 33(2), 507-513. https://doi.org/10 $.4067 / \mathrm{s} 0717-95022015000200016$

Pedraza, O., Salazar, A., Sierra, F., Soler, D., Castro, J., Castillo, P., ... Piñeros, C. (2016). Confiabilidad, validez de criterio y discriminante del Montreal Cognitive Assessment (MoCA) test, en un grupo de adultos de Bogotá. Acta Medica Colombiana, 41, 221-228. https://doi.org/10.36104/amc.2016.693

Pedrosa, I., Suárez, J., y García, E. (2013). Evidencias sobre la Validez de Contenido: Avances Teóricos y Métodos para su Estimación. Acción Psicológica, 10(2), 4-11. http://scielo.isciii.es/pdf/acp/v10n2/02monografico2.pdf

Rendón, P., Aguirre, E., Noroña, L., Betancourt, E. y Vaca, M. (2017). Beneficios de la natación en el asma. Revista Cubana de Investigaciones Biomédicas, 36(2), 150-158. http://www.revibiomedica.sld.cu/index.php/ibi/article /view/11

Ruiz, L. (2017). Competencia motriz acuática: Una cuestión de edades. Revista de Investigación en Actividades Acuáticas, 1(3), 16-22. 
Sanz, I.; Martínez de Haro, V.; Cid, L. (2016). ¿Influye la especialización en los estilos de natación sobre la extensibilidad isquiosural?. International Journal of Medicine and Science of Physical Activity and Sport, 16(61), 55-69. https://www.redalyc.org/pdf/542/54244745005.pdf

Sagarra, L., Gallego, A., y Monroy, A. (2017). Habilidades y destrezas básicas en el medio acuático. Madrid: Paraninfo.

Sánchez-Lastra, M. A., Martínez-Lemos, R. I., Díaz, R., Villanueva, M. y Ayán, C. (2020). Efecto de un programa de natación en la condición física de preescolares. Retos, 37(37), 48-53. https://doi.org/10.47197/retos.v37i37.6 9504.

Véliz, C., Maureira, F. y Rodríguez, M. (2020). Relación de la fuerza, potencia y composición corporal con el rendimiento deportivo en nadadores jóvenes de la Región Metropolitana de Chile. Retos, 38(38), 300-305. htt ps://doi.org/10.47197/retos.v38i38.75638. 